\title{
THE LATTICE OF SUBMODULES OF A MODULE OVER A NONCOMMUTATIVE RING
}

\author{
BY \\ EDMUND H. FELLER
}

Introduction and summary. Of fundamental importance to the theory of ideals in commutative rings satisfying the ascending chain condition for ideals has been the works of Noether [6] and Krull [4]. What follows, in the first two sections, is an attempt to extend more of the Krull-Noether theory of commutative rings to one-sided ideals in noncommutative rings. A decomposition theory for one-sided ideals in the noncommutative case was provided by Fitting [1]. Although Fitting was able to prove uniqueness theorems in certain cases, he did not prove uniqueness theorems for arbitrary noncommutative rings satisfying the ascending chain condition for right ideals. By methods based on the works of Noether and Fitting, we shall prove decomposition and uniqueness theorems for arbitrary noncommutative rings with identity that satisfy the ascending chain condition for right ideals. Actually, the theorems in this paper are proved for what we define to be A-R modules, which is a generalization of noncommutative rings.

In the third section, we discuss completely indecomposable $\mathrm{A}-\mathrm{R}$ modules, which follow along the lines of a paper by Snapper [7]. A sufficient condition for two faithful representations of a noncommutative ring with identity to be equivalent follows from this discussion.

The author is indebted to Professor C. W. Curtis for valuable suggestions in the preparation of this paper.

1. A property of the A.C.C. for $A-R$ modules. We begin by defining an $A-R$ module

Definition 1.1. An $A-R$ module is a system consisting of an additive abelian group $M$, a ring $A$ containing the identity element 1 , a ring $R$ containing the identity element $e$, and two functions defined on the product sets $(M \times A)$ and $(M \times R)$ having values in $M$, such that if $x a$ and $y \alpha$ denote the elements in $M$ determined by the elements $x, y$ in $M, a$ in $A$, and $\alpha$ in $R$, then

$$
x(a+b)=x a+x b,
$$

I-2.

$$
x(a b)=(x a) b,
$$

I-3.

$$
x(\alpha+\beta)=x \alpha+x \beta,
$$

I-4.

$$
x(\alpha \beta)=(x \alpha) \beta,
$$

I-5.

$$
(x+y) a=x a+y a,
$$

Received by the editors March 15, 1955. 
I-6.

I-7.

I-8.

$$
\begin{aligned}
(x+y) \alpha & =x \alpha+y \alpha, \\
x 1 & =x e=x, \\
(x a) \alpha & =(x \alpha) a
\end{aligned}
$$

hold for any $a, b$ in $A$ and $\alpha, \beta$ in $R$.

Definition 1.2. A subgroup $N$ of $M$ is said to be an $R$-submodule of $M$ if $x \alpha \in M$ for every $x$ in $N$ and every $\alpha$ in $R$. A subgroup $N$ of $M$ is said to be an $A$ submodule of $M$ if $x a \in N$ for every $x$ in $N$ and every $a$ in $A$. A subgroup $N$ is an $A-R$ submodule if it is both an $A$ and $R$ submodule.

ExAmpLEs. Every ring with identity can be considered as an $A-R$ module by taking the ring of right multiplications as $R$ and the ring of left multiplications as $A$.

A module $M$ over a ring $R$ containing the identity element can be handled as an $A-R$ module by taking as $A$ the ring of endomorphisms that commute with the endomorphisms which are the multiplications of the elements in $M$ by the elements of $R$.

Conventions. In the future, except where the contrary is stated, the letters $N, N_{1}, N_{2}$, etc., will stand for $R$ and $A$ submodules; the letters $a, b, c$, for elements of $A$; the letters $\alpha, \beta, \gamma$, for elements of $R$; the symbols $\mathfrak{m}, \mathfrak{n}, \mathfrak{p}, \mathfrak{q}$, will stand for ideals in $R$ and $A$.

The proofs of the theorems, except for Theorems 1.2 and 2.2, in the first two sections follow closely the proofs of similar theorems for the commutative case given by Noether and Krull, and are therefore omitted.

For proof of the following theorem we refer to [2, p. 242].

THEOREM 1.1. Let $G_{1}, G_{2}, \cdots, G_{n}$ be normal $N$ subgroups of the group $G$ with operators $N$. If the $A$.C.C. holds for $N$ subgroups of $G / G_{i}, i=1,2, \cdots, n$, then the A.C.C. holds for $N$ subgroups of $G / G_{1} \cap \cdots \cap G_{n}$.

Theorem 1.2. If $M$ is an $A-R$ module, which satisfies the A.C.C. for $A$ submodules and $R$ submodules, then the rings $A / \mathfrak{q}$ and $R / q^{\prime}$ satisfy the A.C.C. for right ideals, where $\mathfrak{q}$ and $\mathfrak{q}^{\prime}$ are the annihilating ideals of $M$ in $A$ and $R$ respectively.

Proof. We shall show that the A.C.C. holds for right ideals in $A / q$. A similar discussion proves this statement for $R / \mathfrak{q}^{\prime}$.

Consider $A$ as a right module over its ring of right multiplications. For a fixed $x_{i}$ in $M$ the set $\left(0: x_{i} ; A\right)=\left\{a \mid x_{i} a=0, x_{i} \in M, a \in A\right\}$ is a right ideal of $A$, and hence an $A$ submodule of $A$. The mapping $\psi$, which takes $a+\left(0: x_{i} ; A\right)$ into $x_{i} a$, is an $A$-isomorphism of the $A$ module $A /\left(0: x_{i} ; A\right)$ upon $x_{i} A$ $=\left\{x_{i} a \mid a \in A\right\}$, which is an $A$ submodule of $M$. Since $x_{i} A$ is an $A$ submodule of $M$, it satisfies the A.C.C. for $A$ submodules, i.e., right ideals. We have also assumed that $M$ satisfies the A.C.C. for $R$ submodules. Hence there must exist a set of generators $x_{1}, x_{2}, \cdots, x_{n}$, for $M$ as an $R$ module. Then, 
from $\mathfrak{q}=(0: M ; A)=\left(0:\left\{x_{1}, \cdots, x_{n}\right\} ; A\right)=\left(0: x_{1} ; A\right) \cap \cdots \cap\left(0: x_{n} ; A\right)$ and Theorem 1.1, it follows that $A / \mathfrak{q}$ satisfies the A.C.C. for right ideals.

2. Decomposition of submodules of an $A-R$ module. Let $M$ be an $A-R$ module.

Definition 2.1. If $N$ is an $R$ submodule of $M$, the set $V^{*}(N)=\{a \mid N a \subseteq N$, $a \in A\}$ is called the centralizer of $N$.

$V^{*}(N)$ is a subring of $A$ which contains the identity element. We denote an arbitrary subring of $V^{*}(N)$ that contains the identity element by $V(N)$.

Definition 2.2. If $T$ is a set in $M$, and $N$ is an $R$ submodule of $M$, then $(N: T ; V)=\{a \mid T a \subseteq N, a \in V(N)\}$ is a right ideal of $V(N)$. If now $T=M$, this set is an ideal of $V(N)$ and will be called the $V$ shadow of $N$. In general we shall denote the $V$ shadow of $N$ by $\mathfrak{n}$.

Definition 2.3. If $N$ is an $R$ submodule of $M$ and $H$ is a set in $V(N)$, then $(N: H)=\{x \mid x h \in N, x \in M$, all $h \in H\}$ is an $R$ submodule.

Definition 2.4. The $V$ radical of an $R$ submodule $N$ of $M$ is defined to be the set of all $a \in V(N)$ such that $a^{t}$ is contained in the $V$ shadow $\mathfrak{n}$ of $N$ for some positive integer $t$.

Definition 2.5. An $R$ submodule $N$ is said to be $V$ primary provided that $x a \in N$, for $x \notin N$ and $a \in V(N)$, implies $M a^{t} \subseteq N$ for some positive integer $t$, i.e., $a$ is contained in the $V$ radical of $N$.

One should note here that if ar $R$ module $N$ is $V$ primary, then it is necessarily $V^{\prime}$ primary, where $V^{\prime}$ is any subring of $V(N)$ containing the identity element.

Definition 2.6. An ideal $\mathfrak{p}$ of an arbitrary ring $K$ will called completely prime provided $a b \in \mathfrak{p}, a, b \in K$, implies at least one of these elements is contained in $\mathfrak{p}$.

Definition 2.7. An ideal $\mathfrak{q}$ of an arbitrary ring $K$ is said to be right primary provided $a b \in \mathfrak{q}, a \notin \mathfrak{q}, a, b \in K$, implies $b^{\mathfrak{s}} \in \mathfrak{q}$ for some positive integer $s$.

One can easily show that the $V$ shadow of a $V$ primary $R$ submodule is a right primary ideal of $A$.

Definition 2.8. An $R$ submodule is irreducible provided it is not the intersection of two $R$ submodules that properly contain it.

THeorem 2.1. If $M$ is an $A-R$ module satisfying the A.C.C. for $R$ submodules, then every irreducible $R$ submodule $N$ is $V(N)$ primary.

TheoRem 2.2. The $V$ radical of a $V$ primary and irreducible $R$ submodule is a completely prime two-sided ideal of $V(N)$.

Proof. The $V$ radical $\mathfrak{p}$ of $N$ is closed under addition. For $a, b \in \mathfrak{p}$, let $s$ and $t$ be the least positive integers such that $a^{s}$ and $b^{t}$ are contained in the $V$ shadow $\mathfrak{n}$ of $N$. Suppose $s>1$ and $t>1$. Then, since $\left(M a^{s-1}\right) a \subseteq N$ and $M a^{s-1} \nsubseteq N$, we have $N \subset(N: a)$, and similarly $N \subset(N: b)$. Suppose $a+b \notin \mathfrak{p}$. 
Then, since $[(N: a) \cap(N: b)] \cdot(a+b) \subseteq N$ and $N$ is $V$ primary, it follows that $(N: a) \cap(N: b) \subseteq N$. However, this is impossible since this would imply that $N$ is reducible, i.e., $N=(N: a) \cap(N: b)$. If $s=1$, then $(a+b)^{t} \in \mathfrak{n}$, and similarly, if $t=1$, then $(a+b)^{s} \in \mathfrak{n}$.

The radical $p$ is closed under right and left multiplication by elements of $V(N)$. Again, for $a \in \mathfrak{p}$, let $s$ be the least positive integer such that $a^{s} \in \mathfrak{n}$. Since $\mathfrak{n}$ is an ideal of $V(N), a^{s} c \in \mathfrak{n}$, for any $c \in V(N)$. If $s>1$, then $a^{s-1}(a c) \in \mathfrak{n}$. Hence, since the $V$ shadow $\mathfrak{n}$ of $N$ is right primary and $a^{s-1} \notin \mathfrak{n}$, it follows $a c \in \mathfrak{p}$. If $s=1$, the result is trivial. Since $(c a)^{s}=c(a c)^{s-1} a, c a \in \mathfrak{p}$.

We conclude by showing that $p$ is completely prime in $V(N)$. Suppose $a_{1} \cdot a_{2} \in \mathfrak{p}, a_{1}, a_{2} \in V(N)$. Then $\left(a_{1} a_{2}\right)^{t} \in \mathfrak{n}$ for some positive integer $t$. From these elements let $a_{i_{1}}, a_{i_{2}}, \cdots, a_{i_{k}}$ be the smallest subcollection whose product $a_{i_{1}} \cdot a_{i_{2}} \cdots \cdots a_{i_{k}}$ is in $\mathfrak{n}$. Since $a_{i_{1}} \cdots a_{i_{k-1}}$ is not in $\mathfrak{n}$ and $\mathfrak{n}$ is right primary, it follows $a_{i_{k}} \in \mathfrak{p}$.

Usual lattice theoretic methods give us the following theorem.

Theorem 2.3. If $M$ is an A-R module satisfying the A.C.C. for $R$ submodules, every $R$ submodule can be expressed as the intersection of a finite number of irreducible $R$ submodules.

Definition 2.9. The intersection $N=N_{1} \cap \cdots \cap N_{t}$ of $R$ submodules is said to be irredundant if $N \subset N_{1} \cap \cdots \cap N_{i-1} \cap N_{i+1} \cap \cdots \cap N, i=1$, $2, \cdots, t$.

Theorem 2.4. If $N=N_{1} \cap \cdots \cap N_{s}=N_{i}^{\#} \cap \cdots \cap N_{s}^{\#}$, where $N_{i}$, $N_{i}^{\#}$, are irreducible, and if these intersections are irredundant, then:

(i) For any $i, 1 \leqq i \leqq s$, there is a $j, 1 \leqq j \leqq s^{\#}$, such that

$$
N=N_{1} \cap \cdots \cap N_{i-1} \cap N_{i}^{\#} \cap N_{i+1} \cap \cdots \cap N_{s} .
$$

(ii) $s=s^{\#}$.

For proof we refer to $[2$, p. 252].

Definition 2.10. If $N$ is an $R$ submodule of $M$, then $N^{\prime}$ is a component element of $N^{\prime}$ if it appears in any irredundant representation of $N$ as the intersection of irreducible $R$ submodules.

Definition 2.11. The ring $F^{*}(N)=\bigcap_{\alpha} V^{*}\left(N_{\alpha}\right)$, where $N_{\alpha}$ ranges over the entire set of component elements of $N$, is called the subcentralizer of $N$.

The subcentralizer $F^{*}(N)$ is contained in the centralizer of $N$ and is not zero since it contains the identity element. We denote an arbitrary subring of $F^{*}(N)$ that contains the identity element by $F(N)$. The radicals, which we shall now consider, will be in just such a subring $F(N)$.

THEOREM 2.5. Let $N=N_{1} \cap \cdots \cap N_{\mathrm{s}}=N_{1}^{\#} \cap \cdots \cap N_{s}^{\#}$ be irredundant intersections where the $N_{i}, N_{i}^{\#}$ are irreducible $R$ submodules, $i=1,2, \cdots, s$. Then the set of distinct $F$ radicals of $N_{\mathrm{r}}, \cdots, N_{s}$ is identical with the set of distinct $F$ radicals of $N_{1}^{\#}, \cdots, N_{s}^{\#}$ in $F(N)$. 
THEOREM 2.6. Let $N_{1}, \cdots, N_{r}$ be irreducible $V^{\prime}$ primary $R$ modules, where $V^{\prime}$ is any subring of $V^{*}\left(N_{1}\right) \cap \cdots \cap V^{*}\left(N_{r}\right)$ containing the identity element. If $N_{i}$ has $V^{\prime}$ radical $\mathfrak{p}$ for all $i$, then $N=N_{1} \cap \cdots \cap N_{r}$ is again $V^{\prime}$ primary with radical $p$. If the intersection $N=N_{1} \cap \cdots \cap N_{r}$ is irredundant and $N$ has radical $\mathfrak{p}_{i}, i=1,2, \cdots, r$, where $\mathfrak{p}_{i} \neq \mathfrak{p}_{j}$, for $i \neq j$, then $N$ is not $V^{\prime}$. primary.

From the preceding theorems, we can state a theorem quite similar to that given by Noether and Krull. However the radicals will not be ideals of the ring $A$, but will be ideals in a subring of $A$. In the example following we show that the same result cannot be stated unless the ring $F(N)$ is used.

Theorem 2.7. Let $M$ be an $A-R$ module satisfying the A.C.C. for $R$ submodules. Let $N$ be an $R$ submodule and $F(N)$ a subring of the subcentralizer of $N$ containing the identity element. Then there exists $F$ primary $R$ submodules $N_{1}, \cdots, N_{s}$, with distinct $F$ radicals $\mathfrak{p}_{1}, \cdots, \mathfrak{p}_{s}$, such that $N=N_{1} \cap \cdots \cap N_{s}$, where this intersection is irredundant. If $N=N_{1}^{\#} \cap \cdots \cap N_{r}^{\#}$ is another such reduction of $N$, then $r=s$, and for a suitable rearrangement of the subscripts the corresponding prime $F$ radicals are equal.

We shall call such a reduction of $N$ an $F$-short representation of $N$, and the prime radicals the $F$ associated prime ideals of $N$.

If the $A-R$ module $M$ satisfies the A.C.C. for $R$ submodules, then for any $R$ submodule $N$ and any ideal $\mathfrak{q}$ of $V(N)$, the $R$ submodules $(N: \mathfrak{q})$ are fixed for large $r$. We denote this limit by $N(\mathfrak{q})$.

Lemma 1. If $M$ satisfies the A.C.C. for $R$ submodules and $N=N_{1} \cap N_{2}$, where $N_{1}$ and $N_{2}$ are $R$ submodules, and $\mathrm{q}$ is an ideal of $V^{\prime}$, which is any subring of $V^{*}\left(N_{1}\right) \cap V^{*}\left(N_{2}\right)$ containing 1 , then $\left(N_{1} \cap N_{2}\right)(\mathfrak{q})=N_{1}(\mathfrak{q}) \cap N_{2}(\mathfrak{q})$.

Lemma 2. If $N$ is a $V$ primary $R$ submodule of $M$, with $V$ radical ideal $\mathfrak{p}$, such that $\mathrm{p}^{r} \subseteq n$, the $V$ shadow of $N$, then

(i) $N(\mathfrak{q})=N$, if $\mathfrak{q} \Phi \mathfrak{p}$,

(ii) $N(\mathfrak{q})=M$, if $\mathfrak{q} \subseteq \mathfrak{p}$,

for an ideal q contained in $V(N)$.

From the preceding lemmas the following theorem is immediate.

THEOREM 2.8. If $M$ satisfies the A.C.C. for $R$ submodules and $N=N_{1} \cap \ldots$ $\cap N_{8}$ is an $F$-short representation of $N$ with distinct $F$ radicals $\left\{p_{i}\right\}, i=1,2, \cdots$, $s$, such that $\mathfrak{p}_{i}^{r_{i}} \subseteq \mathfrak{n}_{i}$ for some positive integers $r_{i}, i=1,2, \cdots, s$, and $q$ is an ideal of $F(N)$ contained in $\mathfrak{p}_{m+1}, \cdots, \mathfrak{p}_{s}$, but not in $\mathfrak{p}_{1}, \cdots, \mathfrak{p}_{m}$, then $N(q)=N_{1} \cap$ $\cdots \cap N_{m}$.

Definition 2.12. If $N=N_{1} \cap \cdots \cap N_{s}$ is an $F$-short representation of $N$, we call $N^{\prime}=N_{i_{1}} \cap \cdots \cap N_{i_{r}}$ an $F$ isolated component of $N$, if no one of the prime ideals $\mathfrak{p}_{i_{1}}, \cdots, \mathfrak{p}_{i_{r}}$ contains an $F$ associated prime ideal of $N$ which is not an ideal of this set. 
Theorem 2.9. If $M$ is an $A-R$ module satisfying the A.C.C. for $R$ submodules and $N=N_{1} \cap \cdots \cap N_{r}=N_{1}^{\#} \cap \cdots \cap N_{s}^{\sharp}$ are two F-short representations of $N$ with distinct $F$ radicals $\mathfrak{p}_{1}, \cdots, \mathfrak{p}_{8}$, such that $\mathfrak{p}_{i}^{r_{i}} \subseteq \mathfrak{n}_{i}$ and $\mathfrak{p}_{i}^{b_{i}} \subseteq \mathfrak{n}_{i}^{\#}$ for positive integers $r_{i}$ and $t_{i}$, where $\mathfrak{n}_{i}$ and $\mathfrak{n}_{i}^{\#}$ are the corresponding $F$ shadows, $i=1,2, \cdots, s$, then the corresponding isolated components are equal.

One may ask in what cases, as in Theorem 2.9, would $p_{i}^{r} \subseteq \mathfrak{n}$ If $M$ satisfies the A.C.C. for $R$ and $F(N)$ submodules, then this is true. For it follows from Theorem 1.2 that $F(N) / \mathfrak{q}$, where $\mathfrak{q}$ is the annihilating ideal of $M$ in $F(N)$, satisfies the A.C.C. for right ideals. Therefore $F(N) / \mathfrak{n}_{i}$ satisfies the A.C.C. for right ideals. Since $\mathfrak{p}_{i}$ is the set of all nilpotent elements in $F(N) / \mathfrak{n}_{i}$ it follows $\mathfrak{p}_{i}^{r} \subseteq \mathfrak{n}$ (see [5]). In addition, if $F(N)$ satisfies the D.C.C. for right ideals, then $\mathfrak{p}_{i}^{r} \subseteq \mathfrak{n}$. In fact, in this case, $F(N) / \mathfrak{p}_{i}$ is a division ring, and the $\mathfrak{p}_{i}$ are maximal ideals of $F(N)$. Therefore each $N_{i}$ would be an isolated component. Hence, in this case, the representation would be unique.

$2^{\prime}$. Example. Let $D$ be a Noetherian ring with identity 1 . Consider $D_{2}$, the ring of $2 \times 2$ matrices with elements in $D$. Then by taking as $R$ the ring of right multiplications and as $A$ the ring of left multiplications, we have an $A-R$ module.

Denote by

$$
\left(\begin{array}{l}
D \\
I
\end{array}\right)
$$

the set of all matrices $\left(a_{i j}\right)$ in $D$ with $a_{11}, a_{12} \in D ; a_{21}, a_{22} \in I$, where $I$ is an ideal in $D$. Then

$$
\left(\begin{array}{l}
D \\
I
\end{array}\right)
$$

is an $R$ submodule.

The proof of the following lemma we leave as an exercise.

Lemma 1.

$$
\left(\begin{array}{l}
D \\
I
\end{array}\right)=\left(\begin{array}{c}
D \\
I_{1}
\end{array}\right) \cap\left(\begin{array}{c}
D \\
I_{2}
\end{array}\right)
$$

if and only if $I=I_{1} \cap I_{2}$.

Denote by $\left(I_{1}, I_{2}\right)_{l}$ the set of all matrices $\left(a_{i j}\right)_{l}$ in $A$ with $a_{11}, a_{12} \in D$, $a_{21} \in I_{1}, a_{22} \in I_{2}$, where $I_{1}$ and $I_{2}$ are ideals in $D$.

THEOREM $2^{\prime}$.1. If

$$
N=\left(\begin{array}{c}
D \\
I
\end{array}\right)
$$

then $V^{*}(N)$ is $(I, D)_{l}$. 
Proof. Certainly this set is contained in the centralizer of $N$. Suppose $\left(a_{i j}\right)_{l} \notin(I, D)_{l}$, i.e., $a_{21} \notin I$. Then for

$$
\left(\begin{array}{ll}
1 & 0 \\
0 & 0
\end{array}\right) \in N
$$

we have

$$
\left(\begin{array}{ll}
a_{11} & a_{12} \\
a_{21} & a_{22}
\end{array}\right)\left(\begin{array}{ll}
1 & 0 \\
0 & 0
\end{array}\right)=\left(\begin{array}{ll}
a_{11} & 0 \\
a_{21} & 0
\end{array}\right) \notin N .
$$

THEOREM $2^{\prime}$.2. If

$$
N=\left(\begin{array}{c}
D \\
I
\end{array}\right)
$$

then the $V^{*}(N)$ shadow of $N$ will be $(I, I)_{l}$, and the $V^{*}(N)$ radical of $N$ will be $(I, p)_{l}$, where $p$ is the radical of $I$ in $D$.

Proof. Certainly $(I, I)_{l}$ is contained in the $V^{*}(N)$ shadow of $N$. If now $\left(a_{i j}\right)_{l} \in V^{*}(N)$ but not contained in $(I, I)_{l}$, i.e., $a_{22} \notin I$. Then for

$$
\left(\begin{array}{ll}
0 & 0 \\
0 & 1
\end{array}\right) \in M
$$

we have

$$
\left(\begin{array}{ll}
a_{11} & a_{12} \\
a_{21} & a_{22}
\end{array}\right)\left(\begin{array}{ll}
0 & 0 \\
0 & 1
\end{array}\right)=\left(\begin{array}{ll}
0 & a_{12} \\
0 & a_{22}
\end{array}\right) \notin N .
$$

If $\left(a_{i j}\right)_{l} \in V^{*}(N)$, then $\left(a_{i j}\right)_{l}=\left(b_{i j}\right)_{l}$, where $b_{11}, b_{12} \in D, b_{21} \in I, b_{22}=c+a_{22}^{t}$ for $c \in I$. Hence, $\left(a_{i j}\right)_{l}$ is contained in the $V^{*}(N)$ shadow if and only if $a_{22} \in p$.

TheOREM $2^{\prime}$.3. If

$$
N=\left(\begin{array}{l}
D \\
I
\end{array}\right)
$$

where $I$ is a primary ideal in $D$, then $N$ is $V^{*}(N)$ primary.

Proof. Suppose $\left(a_{i j}\right)$ is contained in $M$ and is not contained in $N$, say $a_{21} \notin I$. Let $\left(b_{i j}\right)_{l} \in V^{*}(N)=(I, D)_{l}$, i.e., $b_{21} \in I$. Then if $\left(a_{i j}\right)\left(b_{i j}\right)_{l}=\left(b_{i j}\right)\left(a_{i j}\right)$. $=\left(c_{i j}\right) \in N$, we have $c_{21}=d+a_{21} b_{22}$, with $d \in I$. Since $\left(c_{i j}\right) \in N, c_{21}=d+a_{21} b_{22} \in I$. Inasmuch as $I$ is primary in $D$ and $a_{21} \notin I$, then $b_{22} \in p$, the radical of $I$ in $D$. Hence $\left(b_{i j}\right)_{l} \in(I, p)_{l}$, the $V^{*}(N)$ radical of $N$.

A similar discussion holds if $a_{22} \notin I$.

Let $D=F[1, x, y]$, the ring of polynomials in $x$ and $y$ over the field $F$. Let $I=\left(x^{2}, x y\right), I_{1}=(x)$, and $I_{\alpha}=\left(x^{2}, y+\alpha x\right)$, where $\alpha \in F$ and $I_{\alpha} \neq I_{\beta}$ if $\alpha \neq \beta$. Then in $D, I=I_{1} \cap I_{\alpha}$, where the radical $p=(x, y)$ of $I_{\alpha}$ is the same for each 
decomposition of $I$ in primary ideals.

In $D_{2}$, let

$$
N=\left(\begin{array}{c}
D \\
I
\end{array}\right), \quad N_{1}=\left(\begin{array}{c}
D \\
I_{1}
\end{array}\right), \quad \text { and } \quad N_{\alpha}=\left(\begin{array}{c}
D \\
I_{\alpha}
\end{array}\right) .
$$

Then, by Lemma 1 ,

$$
N=N_{1} \cap N_{\alpha} .
$$

However, here the $V^{*}\left(N_{\alpha}\right)$ radicals $\left(N_{\alpha}, p\right)$ are different for each decomposition. If we let $F=\cap I_{\alpha} \cap I_{1}$, then in $(F, D)=\bigcap_{\alpha} V^{*}\left(N_{\alpha}\right) \cap V^{*}\left(N_{1}\right)$ the radical $(F, p)_{l}$ is the same for each decomposition.

Thus although the corresponding $V^{*}$ radicals may be distinct for two decompositions of an $R$ submodule, the corresponding $F^{*}$ radicals are the same.

3. Completely indecomposable $A-R$ modules.

Definition 3.1. An $R$ submodule of an $A-R$ module $M$ is said to be $i n$ decomposable if it can not be expressed as the direct sum of $R$ submodules. An $A$ submodule of $M$ is said to be indecomposable if it can not be expressed as the direct sum of $A$ submodules.

Definition 3.2. A completely indecomposable $A-R$ module is an $A-R$ module where the following four conditions are satisfied.

II-1. Every $R$ submodule of $M$ is indecomposable.

II-2. Every $A$ submodule of $M$ is indecomposable.

II-3. $M$ satisfies the A.C.C. for $A$ submodules and $R$ submodules.

II-4. $M$ satisfies the D.C.C. for $A$ submodules and $R$ submodules.

Definition 3.3. A simple or minimal $R$ submodule is an $R$ submodule $N$ such that if $0 \subseteq N^{\prime} \subseteq N$, where $N^{\prime}$ is an $R$ submodule, then either $0=N^{\prime}$ or $N=N^{\prime}$. A simple or minimal $A$ submodule is defined in the same manner.

From II-3 and Theorem 1.2 we conclude that $R / \mathfrak{q}^{\prime}$ and $R / \mathfrak{q}$ both satisfy the A.C.C. for right ideals, where $\mathfrak{q}^{\prime}$ and $q$ are the annihilating ideals of $M$ in $R$ and $A$ respectively. By II- 1 the 0 module is irreducible as an $R$ submodule, for if $N_{1}$ and $N_{2}$ are $R$ submodules such that $0=N_{1} \cap N_{2}$, then $N_{1}+N_{2}$ $=N_{1} \oplus N_{2}$. Hence, by Theorem 2.1 , the 0 submodule is $A=V^{*}(0)$ primary, and, by Theorem 2.2 , the radical $p$ of 0 in $A$ is a completely prime ideal of $A$. Furthermore, since $A / \mathfrak{q}$ satisfies the A.C.C. for right ideals and $\mathfrak{p}$ is a nil ideal in $A / \mathfrak{q}$, then from a theorem proved by Levitski [5], it follows that $p^{s} \subseteq \mathfrak{q}$ for some positive integer $s$. Similarly by II-2, the 0 module is an irreducible $A$ submodule. Hence 0 is $R$ primary and its radical $\mathfrak{p}^{\prime}$ in $R$ is a completely prime ideal in $R$, where $\mathfrak{p}^{\prime} \subseteq \mathfrak{q}^{\prime}$ for some positive integer $r$.

For a set $H$ in $M$ and a set $\mathfrak{m}$ in $A$ denote by $H \mathfrak{m}$ the set of all sums $\sum x_{i} a_{i}$ for $x_{i} \in H$ and $a_{i} \in \mathfrak{m}$. For a set $\mathfrak{m}^{\prime} \subseteq R$ we denote by $H \mathrm{~m}^{\prime}$ the set of all sums $\sum x_{i} \alpha_{i}$ for $x_{i} \in H$ and $\alpha_{i} \in \mathfrak{m}^{\prime}$.

Lemma 1. Every completely indecomposable $A-R$ module $M$ contains a unique minimal $A-R$ submodule. 
Proof. By II-4, $M$ contains a minimal $R$ submodule $N_{0}$, which is necessarily a unique minimal $R$ submodule. For if $N_{1}$ is another such minimal $R$ submodule, then $0=N_{0} \cap N_{1}$. Similarly $M$ contains a unique minimal $A$ submodule $N^{\prime}$.

We shall now show that $N_{0}$ is an $A$ submodule. Let $H(a)$ be the homomorphism $x \rightarrow x a$ of $M$ for $a \in A$. Then $H(a)$ will be an $R$ homomorphism of $N_{0}$ with kernel $N_{0}$ or 0 . If the kernel is $N_{0}$, then $N_{0} a=0$. If the kernel is 0 , then $N_{0} a$ is an isomorphic image of $N_{0}$ and therefore a minimal $R$ submodule. Since $N_{0}$ is the unique minimal $R$ submodule, then $N_{0} a=N_{0}$. In both cases $N_{0} a \subseteq N_{0}$, and therefore $N_{0}$ is an $A$ submodule. Similarly $N^{\prime}$ is an $R$ submodule. Hence it follows that $N_{0} \subseteq N^{\prime}$ and $N^{\prime} \subseteq N_{0}$, and therefore $N_{0}=N^{\prime}$.

Lemma 2. $\left(0: N_{0} ; A\right)$ and $\left(0: N_{0} ; R\right)$ are maximal right ideals in $A$ and $R$ respectively.

Proof. Let $x R=x A=N_{0}$, which is necessarily cyclic. Let $\psi$ be the homomorphism that takes $a$ into $x a$, for all $a \in A$. If we treat $A$ as a right module over its set of right multiplications, then $\psi$ is an $A$ homomorphism of $A$ upon $N_{0}$ with kernel $(0: x ; A)$. Hence $A /(0: x ; A)$ is $A$ isomorphic to $x A$. Since $x A$ is a simple $A$ module, it follows that $(0: x ; A)$ is a maximal right ideal of $A$. From the relation

$$
N_{0}(0: x ; A)=x R(0: x ; A)=x(0: x ; A) R=0
$$

it follows that $(0: x ; A)=\left(0: N_{0} ; A\right)$.

Similarly $\left(0: N_{0} ; R\right)$ is a maximal right ideal in $R$.

Inasmuch as the 0 module of $M$ is $A$ primary, we have $\left(0: N_{0} ; A\right) \subseteq \mathfrak{p}$. Consequently $\mathrm{p}$ is a maximal right ideal in $A$, and since $\mathfrak{p}$ is also a completely prime ideal in $A$, it follows that $A / \mathfrak{p}$ is a division ring. Similarly $R / \mathfrak{p}^{\prime}$ is a division ring.

Lemma 3. The unique minimal $A-R$ submodule $N_{0}$ is equal to $(0: p)$ and $\left(0: \mathfrak{p}^{\prime}\right)$.

Proof. We have $(0: \mathfrak{p})$ is a vector space over $A / \mathfrak{p}$. If $N_{0} \subset(0: \mathfrak{p})$, then $(0: \mathfrak{p})=N_{0} \oplus N^{\prime}$, where $N^{\prime}$ as an $A / \mathfrak{p}$ subspace. Since $N^{\prime} \subseteq(0: \mathfrak{p})$, it follows that $N^{\prime}$ is an $R$ submodule. This contradicts II-1. Hence $N_{0}=(0: \mathfrak{p})$. Similarly $N_{0}=\left(0: \mathfrak{p}^{\prime}\right)$.

Lemmas 5 and 6 are immediate applications of the following known lemma, which holds true for arbitrary modules over a noncommutative ring.

Lemma 4. Let $M$ be a unitary right module over a ring $R$, where $R$ contains an ideal $p$ such that $M p^{n}=0$ for some integer $n$. If $N$ is a simple $R$ submodule of $M$, then $N p=0$.

Lemma 5. If $M$ is a completely indecomposable $A-R$ module and $N_{1}$ is a maximal $R$ submodule of the $R$ submodule $N_{2}$, then $N_{2} \mathfrak{p}^{\prime} \subseteq N_{1}$, where $\mathfrak{p}^{\prime}$ is the radical of 0 in $R$. 
If we consider $A / \mathfrak{q}$ as a right module over its set of right multiplications, we have the following lemma.

Lemma 6. Let $M$ be a completely indecomposable A-R module. If $\mathfrak{n}_{1}$ and $\mathfrak{n}_{2}$ are right ideals in $A$ containing $\mathfrak{q}$, where $\mathfrak{n}_{1}$ is maximal in the set of right ideals contained in $\mathfrak{n}_{2}$, then $\mathfrak{n}_{2} \mathfrak{p} \subseteq \mathfrak{n}_{1}$ where $\mathfrak{p}$ is the radical of 0 in $A$.

THEOREM 3.1. If $M$ is a completely indecomposable $A-R$ module, then $A / \mathfrak{q}$ satisfies the D.C.C. for right ideals, where $\mathrm{q}$ is the annihilating ideal of $M$ in $A$. Then if

$$
\mathfrak{q} \subset \mathfrak{n}_{0} \subset \mathfrak{n}_{1} \cdots \subset \mathfrak{n}_{\mu}=A
$$

is a composition series of right ideals from $\mathfrak{q}$ to $A$ and

$$
0 \subset N_{0} \subset N_{1} \subset \cdots \subset N_{\lambda}=M
$$

is a composition series of $R$ submodules for $M$, then

$$
0=\left(0: \mathfrak{n}_{\mu}\right) \subset \cdots \subset\left(0: \mathfrak{n}_{0}\right) \subset M
$$

and

$$
\mathrm{q}=\left(0: N_{\lambda} ; A\right) \subset \cdots \subset\left(0: N_{0} ; A\right) \subset A
$$

are composition series of $R$ submodules and right ideals of $M$ and $A / \mathfrak{q}$ respectively.

Proof. Consider $A$ as a right module over its set of right multiplications.

Since $M$ satisfies both chain conditions for $R$ submodules, there exists a chain (2). From this chain we shall show that the factors of (4) are either 0 or isomorphic to a simple $A$ module, i.e., the chain is a normal chain which can be refined to a composition series of right ideals in $A / \mathfrak{q}$. From this we can conclude that $A / \mathfrak{q}$ satisfies the D.C.C. for right ideals, and the length $\mu$ of $A / \mathfrak{q}$ in right ideals is less than or equal to the length $\lambda$ of $M$ in $R$ submodules.

In the second part of this proof we shall show in a similar manner that the chain (3) is a normal chain that can be refined to a composition series of $R$ submodules in $M$. From this we can conclude that $\mu \geqq \lambda$, which completes the proof of the theorem.

Since (2) is a composition series of $M$ in $R$ submodules, we have, by Lemma $5, N_{j} \mathfrak{p}^{\prime} \subseteq N_{j-1}$, where $\mathfrak{p}^{\prime}$ is the radical of 0 in $R$. Hence $N_{j} \mathfrak{p}^{\prime}\left(0: N_{j-1} ; A\right)$ $=N_{j}\left(0: N_{j-1} ; A\right) \mathfrak{p}^{\prime}=0$. From Lemma 3 , we have $\left(0: \mathfrak{p}^{\prime}\right)=N_{0}$, hence $N_{j}\left(0: N_{j-1}\right.$; $A) \subseteq N_{0}$. Since $N_{0}$ is a simple $R$ submodule, it follows that either the $R$ submodule $N_{j}\left(0: N_{j-1} ; A\right)$ equals 0 or $N_{0}$. If this $R$ submodule is zero, we have $\left(0: N_{j-1} ; A\right) /\left(0: N_{j} ; A\right)=0$. Suppose however that this $R$ submodule is equal to $N_{0}$. Then there must exist an $x \in N_{j}, x \notin N_{j-1}$, and an $a \in\left(0: N_{j-1} ; A\right)$ such that $x a \neq 0$. Since $x \in N_{j}, x \in N_{j-1}$, then $N_{j-1}+x R=N_{j}$. It follows, since $x a \neq 0$, that the $A$ submodule $x\left(0: N_{j-1} ; A\right)=\left\{x a \mid a \in\left(0: N_{j-l} ; A\right)\right\}=N_{0}$ since $N_{0}$ is also a minimal $A$ submodule. 
If we treat $A$ as a right module over its set of right multiplications, the correspondence $\psi: a \rightarrow x a$ for $a \in\left(0: N_{j-1} ; A\right)$ is an $A$ homomorphism of $\left(0: N_{j-1} ; A\right)$ onto $N_{0}$ with kernel $\left(0: N_{j-1} ; A\right) \cap(0: x ; A)$, which equals $\left(0: N_{j} ; A\right)$. Hence (4) can be refined to a composition series of $A / \mathfrak{q}$, and $\lambda \geqq \mu$.

We shall now show that $\mu \geqq \lambda$. Since (1) is a composition series we have by Lemma 6 that $\mathfrak{n}_{j} \mathfrak{p} \subseteq \mathfrak{n}_{j-1}$. Hence $\left(0: \mathfrak{n}_{j-1}\right) \mathfrak{n}_{j} \mathfrak{p}=0$, and since $(0: \mathfrak{p})=N_{0}$, it follows that the $A$ submodule $\left(0: \mathfrak{n}_{j-1}\right) \mathfrak{n}_{j}$ equals 0 or $N_{0}$ since $N_{0}$ is a minimal $A$ submodule. If this $A$ module is 0 , we have $\left(0: \mathfrak{n}_{j-1}\right) /\left(0: \mathfrak{n}_{j}\right)=0$. However, if this $A$ submodule is $N_{0}$, there must exist an $a \in \mathfrak{n}_{j}, a \notin \mathfrak{n}_{j-1}$ and an $x \in\left(0: \mathfrak{n}_{j-1}\right)$ such that $x a \neq 0$. Since $a \in \mathfrak{n}_{j}, a \in \mathfrak{n}_{j-1}$ it follows that $\mathfrak{n}_{j-1}+a A=\mathfrak{n}_{j}$. Since $x a \neq 0$, we have $\left(0: \mathfrak{n}_{j-1}\right) a=\left\{x a \mid x \in\left(0: \mathfrak{n}_{j-1}\right)\right\}$ is an $R$ submodule equal to $N_{0}$, since $N_{0}$ is a minimal $R$ submodule. The correspondence $y \rightarrow y a$ for $y \in\left(0: \mathfrak{n}_{j-1}\right)$ is an $R$ homomorphism of $\left(0: \mathfrak{n}_{j-1}\right)$ onto $N_{0}$ with kernel $\left(0: \mathfrak{n}_{j-1}\right)$ $\cap(0: a)=\left(0: \mathfrak{n}_{j}\right)$. Therefore (3) except for repetition is a composition and $\mu \geqq \lambda$.

The following theorem is proved in the same manner as Theorem 3.1.

Theorem 3.2. If $M$ is a completely indecomposable $A-R$ module, then $R / q^{\prime}$ satisfies the D.C.C. for right ideals, where $q^{\prime}$ is the annihilating ideal of $M$ in $R$. In addition, the length of $R / \mathfrak{q}^{\prime}$ in right ideals is equal to the length of $M$ in $A$ submodules.

An immediate application of these theorems gives us the following corollary.

Corollary 1. Let $R$ be a ring with identity that satisfies the A.C.C. and the D.C.C. for both right and left ideals. If no right ideal and no left ideal can be expressed as the direct sum of right ideals and left ideals respectively, then the length of $R$ in left ideals and right ideals is the same.

Lemma 7. If $M$ is a completely indecomposable $A-R$ module, then $[0:(0: \mathfrak{n})$; $A]=\mathfrak{n}$ and $[0:(0: N ; A)]=N$, where $\mathfrak{n}$ is a right ideal in $A$ and $N$ is an $R$ submodule.

Proof. Let $N=N_{j}$ in the composition series

$$
0 \subset N_{0} \subset N_{1} \subset \cdots \subset N_{\mu}=M
$$

of $R$ submodules for $M$. From Theorem 3.1 it follows that

$$
0 \subset\left[0:\left(0: N_{0} ; A\right)\right] \subset \cdots \subset\left[0:\left(0: N_{\mu} ; A\right)\right]=M
$$

is a composition series of $R$ submodules for $M$. Clearly $\left[0:\left(0: N_{i} ; A\right)\right] \subseteq N_{i}$, for all $i$. Since $N_{0}$ is a minimal $R$ submodule, it follows that $N_{0}=\left[0:\left(0: N_{0}\right.\right.$; $A)]$, and this implies that $N_{i}=\left[0:\left(0: N_{i} ; A\right)\right]$ for all $i$. The other part of this lemma is proved in like manner.

A similar lemma can be stated for a right ideal $\mathfrak{n}$ in $R$ and an $A$ submodule 
$N$, by interchanging the letters $R$ and $A$ in the preceding lemma. The following lemma follows immediately.

Lemma 8. In a completely indecomposable $A-R$ module $M$, an $R$ submodule $N$ is completely determined by its annihilating right ideal in $A$, and an $A$ submodule is completely determined by its annihilating right ideal in $R$.

TheOREM 3.3. If $M$ is a completely indecomposable A-R module, then the set $\mathbb{E}$ of $A$ endomorphisms of $M$ consists of the multiplications of the elements of $M$ with the elements of $R$. Hence, if $\mathrm{q}^{\prime}$ is the annihilating ideal of $M$ in $R$, then $\left(\mathbb{S}\right.$ is ring isomorphic with the factor ring $R / q^{\prime}$.

Proof. We shall consider $\mathbb{E}$ not as a ring, but as a module with $R$ as right operator domain, according to the following definitions.

$$
\begin{aligned}
x\left(H_{1}+H_{2}\right) & =x H_{1}+x H_{2}, \\
x(H \alpha) & =(x H) \alpha,
\end{aligned}
$$

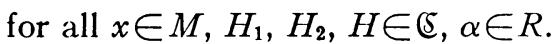

To any $\alpha \in R$ there corresponds the mapping $H(\alpha)$ in $\mathbb{S}$ defined by $x H(\alpha)$ $=x \alpha$ for all $x \in M$. If $R$ is considered as a right module over its set of right multiplications, then the correspondence $\alpha \rightarrow H(\alpha)$ is clearly an $R$ endomorphism from $R$ into $\mathbb{S}$ with kernel $\mathfrak{q}^{\prime}$. Hence $\mathbb{S}$ contains an $R$ submodule $E^{\prime}$ which is $R$ isomorphic to $R / \mathfrak{q}^{\prime}$. We have but to show that $\mathbb{E}=E^{\prime}$. Since $E^{\prime}$ has finite length, namely the length $\mu$ of $R / \mathfrak{q}^{\prime}$ in right ideals, we shall show that $\mathbb{S}$ as an $R$ module has length $\mu$. This would imply that $\mathbb{S}=E^{\prime}$.

By Theorem 3.2, since $R / \mathfrak{q}^{\prime}$ has length $\mu$ for $R$ submodules (right ideals), the length of $M$ for $A$ submodules will be $\mu$. Let

$$
0 \subset N_{0} \subset N_{1} \subset \cdots \subset N_{\mu}=M
$$

be a composition series of $M$ in $A$ submodules. Let $E_{i}$, for $i=0,1, \cdots, \mu$, be the $R$ submodules of $E$ which annihilate $N_{i}$, i.e., $H \in E_{i}$ if $N_{i} H=0$. We shall show that the chain

$$
\text { (ᄃ } \supseteq E_{0} \supseteq E_{1} \supseteq \cdots \supseteq E_{\mu}=0^{\prime} \text {, }
$$

where $0^{\prime}$ denotes the zero-endomorphism, will be a composition series of $R$ submodules for $\mathbb{C}$. This we shall do by showing that $E_{i-1} / E_{i}$ is $R$ isomorphic to the division ring $R / \mathfrak{p}^{\prime}$.

If $H \in E_{i-1}$, then $N_{i} H$ is an $A$ submodule which is homomorphic with $N_{i}$. Since $N_{i-1} H=0$, the length of $N_{i} H$ is at most 1 ; hence $N_{i} H \subseteq N_{0}=\left(0: \mathfrak{p}^{\prime}\right)$. Let $x$ be a fixed nonzero element of $N_{0}$ and $x_{i}$ be a fixed element of $N_{i}$ not in $N_{i-1}$. Then $x R=N_{0}$ and $N_{i-1}+x_{i} A=N_{i}$. Since $N_{i} H \subseteq N_{0}=x R$, there exists an $\alpha \in R$ such that $x_{i} H=x \alpha$. Let $\bar{\alpha}$ denote the coset of $\alpha$ in $R / \mathfrak{p}^{\prime}$. Since $\mathfrak{p}^{\prime}$ is the annihilating ideal of $N_{0}$ in $R$, the correspondence $H \rightarrow \bar{\alpha}$ is an $R$ homomorphism of $E_{i-1}$ into $R / \mathfrak{p}^{\prime}$ with kernel $E_{i}$. Hence $E_{i-1} / E_{i}$ is $R$ isomorphic with an $R$ submodule of $R / \mathfrak{p}^{\prime}$. However, since the only submodules of $R / \mathfrak{p}^{\prime}$ are 0 and 
$R / \mathfrak{p}^{\prime}$ itself, all that remains to show is that $E_{i-1} / E_{i} \neq 0^{\prime}$.

Let $E_{i}^{\prime}$ be the set of all elements $\alpha$ in $R$ such that $N_{i} \alpha=0, i=0,1, \cdots, \mu$. Then by Theorem 3.2, $E_{i-1}^{\#} \supset E_{i}^{\#}$, and consequently there exists an element $\alpha \in R$ such that $N_{i} \alpha \neq 0$ and $N_{i-1} \alpha=0$. From this it follows that $H(\alpha)$ is contained in $E_{i-1}$ and not in $E_{i}$. Hence $E_{i-1} / E_{i} \neq 0^{\prime}$, and therefore (2) is a composition series. Thus $E^{\prime}=\mathbb{C}$.

Corollary 1. Let $M$ be a completely indecomposable $A-R$ module and let $N$ be an $A-R$ submodule. Then every $A$ endomorphism $H^{*}$ of $N$ can be extended to an $A$ endomorphism $H$ of $M$. If $H^{*}$ is an automorphism of $N \neq 0$, then $H$ is an automorphism of $M$.

Proof. If $N$ is an $A-R$ submodule of $M$, then $N$ is a completely indecomposable $A-R$ module. Hence, it follows from Theorem 3.3 that if $H^{*}$ is an $A$ endomorphism, then there exists an element $\alpha \in R$ such that $x H^{*}=x \alpha$ for all $x \in N$. The endomorphism $x H=x \alpha$ for all $x \in M$ is clearly an extension of $H^{*}$ to an $A$ endomorphism $H$ of $M$. If $H^{*}$ is an automorphism of $N \neq 0$, then $\alpha \notin p^{\#}$, where $p^{\#}$ is the radical of the zero-module of $N$ in $R$. If $\mathfrak{p}^{\prime}$ is the radical of the zero-module of $M$ in $R$, clearly $\mathfrak{p}^{\prime} \subseteq \mathfrak{p}^{\#}$, and since $\mathfrak{p}^{\prime}$ is a maximal ideal, then $\mathfrak{p}^{\prime}=\mathfrak{p}^{\#}$. Hence $\alpha \notin \mathfrak{p}^{\prime}$, which implies $H$ is an automorphism of $N$, since $\alpha$ is a unit of $R\left({ }^{1}\right)$.

Lemma 9. If $N_{1}$ and $N_{2}$ are two $R$ submodules of $M$ with respectively the annihilating right ideals $\mathfrak{n}_{1}$ and $\mathfrak{n}_{2}$, then the annihilating right ideal of $N_{1} \cap N_{2}$ is $\mathfrak{n}_{1}+\mathfrak{n}_{2}$.

Proof. Let $N_{3}=N_{1} \cap N_{2}$. Then if $\mathfrak{n}_{3}$ is the annihilating right ideal of $N_{3}$ in $A$, we have, by Lemma $8, \mathfrak{n}_{3}=\left(0: N_{3} ; R\right)$. Since $\left(0: \mathfrak{n}_{1}+\mathfrak{n}_{2}\right)=\left(0: \mathfrak{n}_{1}\right) \cap\left(0: \mathfrak{n}_{2}\right)$ $=N_{1} \cap N_{2}=N_{3}$, then, by Lemma $8,0:\left(0: \mathfrak{n}_{1}+\mathfrak{n}_{2}\right) ; R=\left(0: N_{3} ; R\right)=\mathfrak{n}_{3}$.

The following lemma which holds for arbitrary modules over a noncommutative ring is proved by Snapper $[7$, p. 131].

Lemma 10. Let $M$ and $W$ be two additive groups with the same ring $R$ as operator domain. Let $N_{1}$ and $N_{2}$ be two $R$ submodules of $M$ and let $W_{1}$ and $W_{2}$ be two $R$ submodules of $W$. Let $I_{1}$ be an $R$ isomorphism between $N_{1}$ and $W_{1}$, and let $I_{2}$ be an $R$ isomorphism between $N_{2}$ and $W_{2}$. Let $I_{1}$ map $N_{3}=N_{1} \cap N_{2}$ isomorphically onto $W_{3}=W_{1} \cap W_{2}$, i.e., $I_{1}$ induces an $R$ isomorphism $I_{1}^{*}$ between $W_{3}$ and $N_{3}$. In the same way, let $I_{2}$ map $N_{3}$ isomorphically onto $W_{3}$. Finally, let $I_{1}{ }^{*}=I_{2}{ }^{*}=I_{3}$. Then there exists an $R$ isomorphism between $N_{1}+N_{2}$ and $W_{1}+W_{2}$ which is simultaneously an extension of $I_{1}, I_{2}$ and $I_{3}$.

Definition 3.4. A completely indecomposable $A-R$ module $M$ is said to be $d u o$, provided every $R$ submodule is an $A$ submodule and every $A$ submodule is an $R$ submodule.

(1) It is shown in Jacobson [3, p. 57] that if $R / p$ is a division ring, then the elements not in $p$ are units of $R$, if $\mathfrak{p}$ is a nil-ideal. 
Since the set of annihilators of an $A-R$ submodule in $A$ or $R$ is a two-sided ideal, we have by Theorem 3.2 and Lemma 7

Theorem 3.4. If $M$ is a duo completely indecomposable $A-R$ module, then the length of $M$ in $A-R$ submodules, the length of $A / q$ in two-sided ideals, and the length of $R / q^{\prime}$ in two-sided ideals, where $\mathfrak{q}$ and $\mathfrak{q}^{\prime}$ are the annihilating ideals of $M$ in $A$ and $R$ respectively, are the same. In addition, if $\mathfrak{n}$ is a two-sided ideal of $A$ and $N$ is an $A-R$ submodule of $M$, then

$$
0:(0: \mathfrak{n}) ; A=\mathfrak{n} \text { and } 0:(0: N ; A)=N^{(2)} \text {. }
$$

Lemma 11. Let $M$ be a duo completely indecomposable $A-R$ module. If $M$ has a unique maximal $A-R$ submodule, then $M$ is $R$ isomorphic to $R / \mathfrak{q}^{\prime}$ and $A$ isomorphic to $A / \mathfrak{q}$, where $\mathfrak{q}^{\prime}$ and $\mathfrak{q}$ are the annihilating ideals of $M$ in $R$ and $A$ respectively.

Proof. If $N$ is the unique maximal $A-R$ submodule of $M$, then for $x \in M$, $x \notin N$, it follows that $x R=x A=M$. The mapping $a \rightarrow x a$, all $a \in A$, is an $A$ homomorphism of $A$ onto $M$ with kernel $\mathfrak{q}$, for suppose $x a=0$, then $x a R=0$, which implies that $x R a=0$, i.e., $M a=0$.

The following is an extension of a theorem proved by Snapper [7, p. 133].

Theorem 3.5. Let $M$ be a duo completely indecomposable $A-R$ module and $W$ a duo completely indecomposable $A-K$ module, then $M$ is $A$ isomorphic to $W$ if and only if they have the same annihilating ideals in $A$.

Proof. It is obvious that if $M$ and $W$ are $A$ isomorphic, they have the same annihilating ideals in $A$. Hence we assume that $M$ and $W$ have the same annihilating ideal $q$ in $A$ and prove they are $A$ isomorphic.

Since the length of $M$ and $W$ in $A-R$ submodules are both equal to the length of the difference module $A / \mathfrak{q}$ in $A$ submodules (two sided ideals), let $\mu$ be the common length of $W, M$ and $A / \mathfrak{q}$. If $\mu=1$, both $M$ and $W$ have their zero submodules as unique maximal $A-R$ submodules, and hence, by Lemma 11 , are $A$ isomorphic to $A / \mathfrak{q}$, and hence isomorphic to each other. Consequently, we assume $\mu>1$ and make our induction hypothesis that Theorem 3.5 has been proved for $\mu=1,2, \cdots, \mu-1$. There are two cases to be considered.

CASE 1. One of the two modules, say $M$, has a unique maximal $A-R$ submodule. Since $M$ has a unique maximal submodule, it follows from Lemma 11 that $M$ is $A$ isomorphic to $A / \mathfrak{q}$. This implies that $A / \mathfrak{q}$ contains a unique minimal two sided ideal. Consequently, there exists only one two-sided ideal $q_{1}$ where the length of $A / \mathfrak{q}_{1}$ is $\mu-1$ in two-sided ideals. If $0^{\prime}$ is the zero element of $W$, the length of $W_{1}=\left(0^{\prime}: \mathfrak{q}_{1}\right)$ is $\mu-1$ and hence $W_{1}$ is a maximal $A-R$ submodule of $W$. We assert that $W_{1}$ is the only maximal $A-R$ submodule of $W$.

(2) Notice that this implies that every right ideal of $A$ or $R$ is a two-sided ideal. 
Let $W_{2}$ be any maximal $A-R$ submodule of $W$ with $q_{2}$ as annihilating ideal in $A$. Then, by Theorem 3.4, the length of $W_{2}$ is $\mu-1$, and the length of $A / \mathrm{q}_{2}$ is $\mu-1$. Consequently, $\mathfrak{q}_{1}=\mathfrak{q}_{2}$ and $W_{2}=\left(0^{\prime}: \mathfrak{q}_{2}\right)=\left(0: \mathfrak{q}_{1}\right)=W_{1}$. Lemma 11 then implies that $M$ and $W$ are $A$ isomorphic to $A / \mathfrak{q}$ and hence $W$ and $M$ are $A$ isomorphic.

CASE 2. Both $M$ and $W$ have at least iwo different maximal $A$ submodules. Let $N_{1}$ and $N_{2}$ be two different maximal $A-R$ submodules of $M$, which implies that $M=N_{1}+N_{2}$. The length of $N_{1}$ and $N_{2}$ is $\mu-1$. If $\mathfrak{q}_{1}$ and $\mathfrak{q}_{2}$ are the annihilating ideals in $A$ of $N_{1}$ and $N_{2}$, then $\mathfrak{q}_{1}=\left(0: N_{1} ; A\right)$ and $\mathfrak{q}_{2}=\left(0: N_{2} ; A\right)$. Furthermore, by Theorem 3.4, $q_{1} \neq q_{2}$ and the length of both the difference modules $A / \mathfrak{q}_{1}$ and $A / \mathfrak{q}_{2}$ in $A$ submodules (two-sided ideals) is $\mu-1$. Consider the $A-R$ submodules $W_{1}=\left(0^{\prime}: \mathfrak{q}_{1}\right)$ and $W_{2}=\left(0^{\prime}: \mathfrak{q}_{2}\right)$ of $W$. Then, by Theorem 3.4, $W_{1} \neq W_{2}$, and the length of $W_{1}$ and $W_{2}$ is $\mu-1$, i.e., $W_{1}$ and $W_{2}$ are distinct maximal $A-R$ submodules of $W$, which implies that $W_{1}+W_{2}=W$. Since $N_{1}$ is a duo completely indecomposable $A$-R module and $W_{2}$ is a duo completely indecomposable $A-K$ module with the same annihilating ideal $q_{1}$, it follows by induction that $N_{1}$ and $W_{1}$ are $A$ isomorphic. In the same way $N_{2}$ and $W_{2}$ are $A$ isomorphic. Hence, let $I_{1}$ be the $A$ isomorphism which maps $N_{1}$ onto $W_{1}$ and $I_{2}$ the $A$ isomorphism that maps $N_{2}$ onto $W_{2}$. We claim that $I_{1}$ maps $N_{3}=N_{1} \cap N_{2} A$ isomorphically onto $W_{3}=W_{1} \cap W_{2}$ and that $I_{2}$ maps $N_{3} A$ isomorphically onto $W_{3}$. In the first place, the annihilating ideal of $N_{3}$ and $W_{3}$ in $A$ is $\mathrm{q}_{1}+\mathrm{q}_{2}$ according to Lemma 9. In the second place, both $N_{3} I_{1}$ and $N_{3} I_{2}$ are $A-R$ submodules of $W$ which are $A$ isomorphic with $N_{3}$. Hence these modules have $\mathfrak{q}_{1}+\mathfrak{q}_{2}$ as annihilating ideals in $A$, which implies, according to Theorem 3.4, that $N_{3} I_{1}=N_{3} I_{2}=W_{3}$. Let $I_{1}{ }^{*}$ and $I_{2}{ }^{*}$ be the $A$ isomorphism which map $N_{3}$ onto $W_{3}$ and which are induced respectively by $I_{1}$ and $I_{2}$. However, we cannot as yet apply Lemma 10 since it may be that $I_{1}^{*} \neq I_{2}{ }^{*}$. However, we shall change $I_{1}$ into a new $A$ isomorphism $J_{1}$ such that $J_{1}^{*}=I_{2}^{*}$. Let $\left(I_{1}^{*}\right)^{-1}$ be the inverse of $I_{1}^{*}$; hence $\left(I_{1}^{*}\right)^{-1}$ maps $W_{3} A$ isomorphically onto $N_{3}$. Then $\left(I_{1}^{*}\right)^{-1} I_{2}{ }^{*}$ is clearly an $A$ isomorphism, say $H^{*}$, of $W_{3}$ onto itself. Since $W_{3} \subseteq W_{1}$, it follows from Theorem 3.3, Corollary 1 , that there exists an $A$ isomorphism $H$ of $W_{1}$ onto itself, where $H$ is an extension of $H^{*}$. For $J_{1}$ we then take the $A$ isomorphism $J_{1}=I_{1} H$ which clearly maps $N_{1}$ onto $W_{1}$. Furthermore, if $J_{1}^{*}$ is the $A$ isomorphism between $N_{3}$ and $W_{3}$, induced by $J_{1}$, then $J_{1}^{*}=I_{2}{ }^{*}$ because, if $x \in N_{3}$, then $x J_{1}^{*}=x I_{1} H=x I_{1}^{*} H$ and, since $x I_{1}^{*} \in W_{3}$, then $x I_{1}^{*} H=x I_{1}^{*} H^{*}=x I_{1}^{*}\left(I_{1}^{*}\right)^{-1} I_{2}{ }^{*}=x I_{2}{ }^{*}=x I_{2}$. We can conclude from Lemma 10 that there exists an $A$ isomorphism between $N_{1}+N_{2}=M$ and $W_{1}+W_{2}=W$ which proves our theorem.

In terms of representations this theorem has the following meaning. Let $M$ and $W$ be faithful representation spaces for a ring $A$ with identity, where $\mathfrak{G}_{1}$ and $\mathfrak{C}_{2}$ are the ring of $R$ endomorphisms of $M$ and $W$ respectively. Consider $M$ and $W$ as representation spaces for $\mathfrak{C}_{1}$ and $\mathfrak{C}_{2}$. Then, if every $A$ subspace of $M$ is a $\mathfrak{C}_{1}$ subspace and if every $\mathfrak{E}_{1}$ subspace of $M$ is an $A$ subspace, and if 
every $A$ subspace of $W$ is a $\mathfrak{C}_{2}$ subspace and every $\mathbb{E}_{2}$ subspace of $W$ is an $A$ subspace, and if, in addition, $M$ and $W$ have composition series of $A$ subspaces and unique minimal $A$ subspaces, these representations are equivalent.

$3^{\prime}$. Examples. Examples of completely indecomposable $A-R$ modules where $A$ and $R$ are commutative are given by Snapper in [7].

Example $3^{\prime}$.1. Every Grassmann $\left({ }^{3}\right)$ Algebra with finite basis can be considered as a duo completely indecomposable $A-R$ module by taking as $R$ the ring of right multiplications and as $A$ the ring of left multiplications. Suppose the basis elements are $e_{1}, e_{2}, \cdots, e_{n}$. Then this $A$-R module is duo since $e_{i} e_{j}=-e_{j} e_{i}$, and satisfies II-1 and II-2 since $x R=x A$, where $x=e_{1} \cdot e_{2} \cdots e_{n}$, is a unique minimal $A-R$ submodule (two-sided ideal).

Example $3^{\prime} .2$. Let $F$ be a field and $x_{1}, x_{2}$ and $x_{3}$ be indeterminants. Consider $H=F\left[1, x_{1}, x_{2}, x_{3}\right]$, where the following conditions are satisfied:

(1) Associate law of multiplication.

(2) Two sided distributive law.

(3) $a 1=1 a=a, a x_{i}=x_{i} a, i=1,2,3$, for all $a \in F$.

(4) $x_{i} x_{j} x_{k}=x_{1}, i, j, k=2,3$.

(5) $x_{i} x_{j} x_{k} x_{l}=0, i, j, k, l,=1,2,3$.

$H$ is an algebra with finite bases over $F$, for if $x \in H$, then $x=a_{0}+a_{1} x_{1}+a_{2} x_{2}$ $+a_{3} x_{3}+a_{4} x_{2}^{2}+a_{5} x_{3}^{2}+a_{6} x_{2} x_{3}+a_{7} x_{3} x_{2}$. Properties (4) and (5) imply $0=x_{1}^{2}=x_{1} x_{i}$, $i=2,3$. If we let $R$ be the ring of right multiplications and $A$ be the ring of left multiplications, then $H$ is a completely indecomposable $A$-R module, for $x_{1} R=x_{1} H=H x_{1}=x_{1} A$ is a unique minimal $A$ submodule and a unique minimal $R$ submodule. $H$ is not duo since $x_{2} H=x_{2} R$ is not an $A$ submodule because it does not contain $x_{3} x_{2}$.

\section{BIBLIOGRAPHY}

1. H. Fitting, Primärkomponentenzerlegung in nichtkommutativen Ringe, Math. Ann. vol. 46 (1935).

2. P. M. Grundy, A generalization of additive ideal theory, Proc. Cambridge Philos. Soc. vol. 38 (1941).

3. N. Jacobson, The theory of rings, Mathematical Surveys, no. 2, 1943.

4. W. Krull, Ein neuer Bewies für die Haupsätze der allgemeinen Idealtheorie, Math. Ann. vol. 90 (1923).

5. J. Levitski, On multiplicative systems, Compositio Math. vol. 8 (1950).

6. E. Noether, Idealtheorie in Ringbereichen, Math. Ann. vol. 83 (1921).

7. E. Snapper, Completely indecomposable modules, Canadian Journal of Mathematics vol. 1(1949).

UNIVERSITY OF WISCONSIN, Madison, Wis.

(3) For a discussion of Grassmann Algebras read Theory of Lie groups by C. C. Chevalley. 